\title{
FORRAJE VERDE HIDROPÓNICO DE MAÍZ (Zea mays) DESHIDRATADO EN EL ENGORDE DE CONEJOS NUEVA ZELANDA (Oryctolagus cuniculus)
}

\author{
${ }^{\bullet}$ Adolfo Sánchez Laiño ${ }^{1,2}$, Alejandro Meza Chica², Anthony Álvarez Tubay ${ }^{2}$, Lauden Rizzo Zamora ${ }^{3}$, \\ Ángel Guadalupe Puente ${ }^{3}$ \\ ${ }^{1}$ Unidad de Investigación Cientifica y Tecnológica, Universidad Técnica Estatal de Quevedo, km 7 vía \\ Quevedo - El Empalme, C. P. 73. Mocache, Los Ríos, Ecuador. ${ }^{\triangleright}$ adolsanlai@hotmail.com \\ ${ }^{2}$ Facultad de Ciencias Pecuarias, Universidad Técnica Estatal de Quevedo, $\mathrm{km} 7$ vía a El Empalme, \\ Mocache, Los Ríos, Ecuador. \\ ${ }^{3}$ Unidad de Estudios a Distancias, Universidad Técnica Estatal de Quevedo, km 1 1/2 vía a Santo Domingo \\ de los Tsáchilas, C. P. 73. Quevedo, Los Ríos, Ecuador
}

\begin{abstract}
RESUMeN
$\mathrm{E}^{\prime}$ 1 presente trabajo se ejecutó en el sector El Mamey, ciudad de Babahoyo, Provincia Los Ríos, cuya ubicación geográfica es: $0.1^{\circ}$ de Latitud Sur y $79^{\circ} 32^{\prime \prime}$ de Latitud Central Oeste, a una altura de $5 \mathrm{msnm}$. El trabajo de campo duró 56 días. Los objetivos fueron siguientes: Determinar el incremento de peso en el engorde de conejos Nueva Zelanda bajo el efecto del consumo de Forraje Verde Hidropónico de Maíz (FVHM) y la rentabilidad. Se utilizaron 32 conejos Nueva Zelanda de 45 días de edad, con un peso promedio de 961.70 g. Se aplicó un Diseño Completamente al Azar (DCA) con cuatro repeticiones. Los tratamientos evaluados fueron: T0: Pasto Saboya + balanceado (Testigo); T1: FVHM, deshidratado por 12 horas + balanceado; T2: FVHM, deshidratado por 24 horas + balanceado y T3: FVHM, deshidratado por 36 horas + balanceado. Para las comparaciones entre medias se utilizó la prueba de Tukey $(\mathrm{P} \leq 0.05)$. Para obtener la rentabilidad de los tratamientos se aplicó la Relación Beneficio - Costo. Se evaluó el consumo de FVHM (g), consumo de alimento (g), ganancia de peso (g), conversión de alimento, peso a la canal (g) y rendimiento a la canal (\%). El mayor consumo $(\mathrm{P}<0.05)$ de forraje $\left(14.14-13.94 \mathrm{~g} \mathrm{animal}^{-1}\right.$ día $a^{-1}$ de MS), rendimiento a la canal (55.65 - 53.74\%) y Relación Beneficio Costo más eficiente (0.69 - 0.64) se obtuvo al suministrar FVHM, deshidratado entre 24 y 36 horas más balanceado peletizado. El peso final, la ganancia de peso y la conversión alimenticia no se vieron afectados $(\mathrm{P}>0.05)$.
\end{abstract}

Palabras claves: Conejos, alimentación, forraje verde hidropónico
$\mathrm{T}$ The present work was carried out in The Mamey sector, city of Babahoyo, Los Ríos province, whose geographical location is: $0.1^{\circ}$ of South Latitude and $79^{\circ}$ 32 " of West Central Latitude at a height of 5 meters above sea level. The field work lasted 56 days and the objectives were as follows objectives: To determine the increment of weight in fattening of New Zealand rabbits under the effect of the consumption of Hydroponic Green Maize (HGFC) and its profitability. 32 New Zealand rabbits of 45 days of age were used, with a weight average of $961.7 \mathrm{~g}$. A Complete Randomized Design (CRD) was applied with four replications. The evaluated treatments were: T0: I Saboya pasture + balanced food (Tester); T1: 12 hours dehydrated HGFC + balanced food; T2: 24 hours dehydrated HGFC + balanced food and T3: 36 hours dehydrated HGFC + balanced food. For the comparisons among means, Tukey test was used $(\mathrm{P} \leq 0.05)$. The Relationship Benefit - Cost was applied in order to obtain the treatments profitability. The consumption of HGFC (g) and food (g), gain of weight $(\mathrm{g})$, food conversion, weight $(\mathrm{g})$ and yield to the channel $(\%)$ were evaluated. The biggest $(\mathrm{P}<0.05)$ consumption of forage $(14.14-13.94 \mathrm{~g}$ animal day $^{-1}$ of DM), yield to the channel (55.65 - 53.74\%) and more efficient Relationship Benefit - Cost (0.69 $0.64)$ were obtained when providing dehydrated HGFC between 24 and 36 hours plus balanced food. The final weight, the gain of weight and the nutritious conversion were not affected $(\mathrm{P}>0.05)$.

Key words: Rabbits, feeding, hydroponic green forage

\section{INTRODUCCION}

$\mathrm{P}$ ara optimizar la producción de forraje verde fresco se pueden utilizar técnicas de cultivo hidropónico, considerado como un avance en la producción agrícola; ya que presenta ventajas técnicas, económicas, disminución de espacio, ahorro de energía y labores culturales (Domínguez, 1989; Bungarín et al., 1998).

La hidroponía se define como el cultivo sin suelo sobre sustratos inertes, con el uso de soluciones nutritivas que abastecen los requerimientos nutricionales

Recibido: diciembre, 2010. Aceptado: febrero, 2011.

Publicado como NOTA TECNICA en Ciencia y Tecnología 3(2): 21-23. 2010 de las plantas (Resh, 2001). Con el forraje verde hidropónico (FVH), se puede alimentar a vacunos, caprinos, porcinos, equinos, avestruces y conejos (Bautista y Nava, 2002). La producción cunícola basada en este tipo de alimentación es más económica que cuando se usa exclusivamente balanceado (Reinoso, 1994). La producción de FVH, presenta grandes alternativas para la producción animal, debido al gran rendimiento de materia verde o seca durante 
todo el año, calidad y cantidad de proteína producida en pequeñas áreas, sin necesidad de suelo, maquinaria agrícola y pequeñas cantidades de agua (AGRORED, 2003).

\section{Materiales y Métodos}

$\mathrm{E}$ 1 presente trabajo se ejecutó en el sector El Mamey, ciudad de Babahoyo, Provincia Los Ríos, cuya ubicación geográfica es: $0.1^{\circ}$ de Latitud Sur y $79^{\circ} 32^{\prime}$, de Latitud Central Oeste. La zona tuvo una temperatura de $25.7^{\circ} \mathrm{C}$, una humedad relativa de $77.0 \%$, una precipitación media anual de $1925.4 \mathrm{~mm}$ y una altura de $5 \mathrm{msnm}$. El trabajo de campo duró 56 días. Se utilizaron 32 conejos Nueva Zelanda de 45 días de edad, con un peso promedio de $961.70 \mathrm{~g}$. Se aplicó un diseño completamente al azar (DCA) con cuatro repeticiones. La unidad experimental estuvo conformada por dos animales. Los tratamientos evaluados fueron: T0: Pasto saboya + balanceado (Testigo); T1: FVHM, deshidratado por 12 horas + balanceado; T2: FVHM, deshidratado por 24 horas + balanceado y T3: FVHM, deshidratado por 36 horas + balanceado. Para las comparaciones entre medias se utilizó la prueba de Tukey $(\mathrm{P} \leq 0.05)$. Para obtener la rentabilidad de los tratamientos se utilizó la Relación Beneficio - Costo. Se evaluó el consumo de FVHM (g), consumo de alimento (g), ganancia de peso $(\mathrm{g})$, conversión de alimento, peso a la canal $(\mathrm{g})$ y rendimiento a la canal (\%).

\section{Resultados y Discusión}

L os resultados de las variables evaluadas se detallan $L_{\text {en }}$ los cuadros 1 y 2 . El pasto saboya (Panicum maximun Jack) fue el forraje de mayor consumo $(\mathrm{P}<0.05)$. Sin embargo el mayor consumo $(\mathrm{P}<0.05)$ de
FVHM, lo registraron los tratamientos T2 y T3 (791.86 y $780.71 \mathrm{~g}$, lo que representa un consumo animal día ${ }^{-1}$ de 14.14 y $13.94 \mathrm{~g}$ de MS).

El tratamiento de mayor consumo de alimento $(\mathrm{P}<0,05)$ fue el testigo (T0), sin embargo entre los tratamientos T2 y T3 el consumo de alimento fue similar ( $\mathrm{P}>0.05)$ (3255.90 y $3217.20 \mathrm{~g}$, lo que representa un consumo animal día ${ }^{-1}$ de 58.14 y 57.45 g de MS, respectivamente.

El peso vivo, la ganancia de peso y la conversión alimenticia no se vieron afectadas $(\mathrm{P}>0.05)$ por efecto de los tratamientos. Sin embargo el tratamiento T2, reportó el mayor peso vivo (1909.50 g) y la mayor ganancia de peso $(908.50 \mathrm{~g}$, lo que representa una ganancia animal día $^{-1}$ de $16.22 \mathrm{~g}$ ). Mientras que los tratamientos T3 y T2 reportan la conversión alimenticia más eficiente (3.54 y 3.58). De igual manera estos tratamientos reportan el mayor rendimiento a la canal $(\mathrm{P}<0.05)$ y la mejor relación beneficio costo $(55.65 ; 53.74 \%-0.64$ y 0.69 , respectivamente).

El consumo de FVHM, consumo de alimento y ganancia de peso obtenidos en la presente investigación fueron inferiores a los reportados por Salazar (2004), quien al incluir hasta un $30 \%$ de forraje verde hidropónico henificado de cebada (FVHHC) en dietas para conejos, reportó consumos de 1,329.00; 3,329.00 y una ganancia de $1,089.00 \mathrm{~g}$, respectivamente. Además con los de León (2005), quien reporta consumos y ganancias de $3,510.00$ y $1,190.00 \mathrm{~g}$ al evaluar el efecto de diferentes fotoperiodos y soluciones nutritivas en la producción de FVHM. Sin embargo el peso final, el rendimiento a la canal y la relación beneficio costo fueron superiores a los reportados por el primer autor $(1,872.0 \mathrm{~g} ; 48.82 \%$ y 0.46 , respectivamente). El peso final fue similar al obtenido por Nava et al., (2005), quienes al suministrar $60 \%$ de FVH $+40 \%$ de balanceado comercial, reportaron un peso vivo a las siete semanas pos destete de 1,908.00 gramos.

Cuadro 1. Consumo de forraje (g), consumo de alimento (g), peso inicial (g), peso vivo (g), ganancia de peso (g), conversión alimenticia y rendimiento a la canal (\%), en el engorde de conejos Nueva Zelanda, bajo el efecto del consumo de Forraje Verde Hidropónico de Maíz (FVHM). Babahoyo.

\begin{tabular}{cccccccc}
\hline \multirow{2}{*}{ Tratamiento } & $\begin{array}{c}\text { Consumo } \\
\text { forraje }\end{array}$ & $\begin{array}{c}\text { Consumo } \\
\text { alimento }\end{array}$ & $\begin{array}{c}\text { Peso } \\
\text { inicial }\end{array}$ & $\begin{array}{c}\text { Peso } \\
\text { vivo }\end{array}$ & $\begin{array}{c}\text { Ganancia } \\
\text { peso }\end{array}$ & $\begin{array}{c}\text { Conversión } \\
\text { alimenticia }\end{array}$ & $\begin{array}{c}\text { Rendimiento } \\
\text { canal }\end{array}$ \\
\hline $\mathrm{T} 0$ & $1,615.35 \mathrm{a}$ & $4,040.85 \mathrm{a}$ & 987.50 & $1,775.00$ & 787.50 & 5.13 & $50.13 \mathrm{bc}$ \\
$\mathrm{T} 1$ & $479.21 \mathrm{c}$ & $2,943.21 \mathrm{c}$ & 906.25 & $1,650.00$ & 743.75 & 3.96 & $48.94 \mathrm{c}$ \\
$\mathrm{T} 2$ & $791.86 \mathrm{~b}$ & $3,255.86 \mathrm{~b}$ & $1,000.00$ & $1,909.50$ & 909.50 & 3.58 & $53.74 \mathrm{ab}$ \\
$\mathrm{T} 3$ & $780.72 \mathrm{~b}$ & $3,217.22 \mathrm{~b}$ & 965.75 & $1,875.00$ & 909.25 & 3.54 & $55.65 \quad \mathrm{a}$ \\
\hline
\end{tabular}

*Literales distintos en la misma columna muestran diferencias significativas según Tukey $(\mathrm{P} \leq 0.05)$ 
Cuadro 2. Análisis Económico (USD) de la evaluación del Forraje Verde Hidropónico de Maíz (FVHM) en el engorde de conejos Nueva Zelanda. Babahoyo.

\begin{tabular}{lcccc}
\hline \multirow{2}{*}{ Rubros } & \multicolumn{4}{c}{ Tratamientos } \\
\cline { 2 - 5 } & T0 & T1 & T2 & T3 \\
\hline Total de Egresos. & 37.97 & 37.41 & 37.56 & 37.57 \\
Total de Ingresos. & 56.26 & 47.66 & 63.47 & 61.44 \\
Beneficio Neto. & 18.29 & 10.25 & 25.91 & 23.87 \\
\hline R. B/C. & $\mathbf{0 . 4 8}$ & $\mathbf{0 . 2 7}$ & $\mathbf{0 . 6 9}$ & $\mathbf{0 . 6 4}$ \\
\hline
\end{tabular}

\section{Conclusiones}

I os resultados obtenidos en la presente investigación - nos permiten llegar a las siguientes conclusiones.

El consumo de forraje, ganancia de peso y relación Beneficio Costo, más eficiente, se obtienen al suministrar Forraje Verde Hidropónico de Maíz(FVHM), deshidratado entre 24 y 36 horas, más balanceado peletizado. Cuando el Forraje Verde Hidropónico de Maíz (FVHM) es suministrado con pocas horas de deshidratación (12h) se obtienen bajos rendimientos productivos. El mayor consumo de alimento lo presentó el tratamiento testigo. El mejor rendimiento a la canal se lo obtuvo al suministrar Forraje Verde Hidropónico de Maíz (FVHM), deshidratado entre 36 y 24 horas, más balanceado peletizado.

\section{LiteratuRA CITADA}

AGRORED, A. 2003. Horticultura, Fruticultura, Fertilización y Cultivos Hidropónicos. (en línea). Consultado 14 Oct. 2008. Disponibles en: http: // www. Agrored. Com. Mx/agricultura/63-Forraje html.

Bautista, S; Nava, J. 2002. Producción de Forraje Verde Hidropónico de trigo triticum, tesis de Licenciatura Universal Autónoma de Guerrero (UAG).

Bungarín, M; Baca, C; Martínez, H; Tirado, J. 1998. Amonio/nitrato y concentración iónica total de la solución nutritiva en crisantemo II extracción nutrimental de hojas, Montecillo, México.
Domínguez, V. 1989. Tratado de Fertilización $2^{\mathrm{a}}$ edición. Mundi Prensa.- México.

León, S. 2005. Efecto del Fotoperiodo en la producción de Forraje Verde Hidropónico de Maíz con diferente soluciones nutritivas para alimentación de conejos en el período de engorde. Tesis de grado FCP ESPOCH. pp 51-59.

Nava, J; Córdova, A. 2005. Alimento balanceadoforraje verde hidropónico en la alimentación de conejos criollos (Oryctolagus cuniculus). Revista Electrónica de Veterinaria REDVET. Vol. VI, No 10. (en línea). Consultado 14 Oct. 2008. Disponible en http://www.veterinaria.org/revista/ redvet/n101005.html.

Reinoso, J. 1994. Evaluación de dietas granuladas altas en forraje en la alimentación de conejos. Tesis de Licenciatura, Universidad autónoma, Chapingo México.

Resh, H. 2001. Cultivos hidropónicos; nuevas técnicas de producción. Versión española de José Santos Caffarena. Madrid, España, Ediciones MundiPrensa. 284p.

Salazar, W. 2004. Utilización del Forraje Verde Hidropónico Henificado de Cebada en reemplazo de la alfalfa en la alimentación de conejos, tesis de grado, FCP - ESPOCH. Riobamba Ecuador. pp 45-54. 